\title{
Charting Complex Change: Application of the e-Health Implementation Toolkit (e-HIT) in 'dallas'
}

\author{
Alison M. Devlin, \\ Catherine O' Donnell, Frances Mair, \\ Institute of Health \& Well-Being \\ University of Glasgow \\ FirstName.LastName@glasgow.ac.uk
}

\author{
Marilyn McGee-Lennon, \\ Dept. of Computer \& \\ Information Science, \\ University of Strathclyde \\ FirstName.LastName@strath.ac.uk
}

\begin{abstract}
The 'dallas' (Delivering Assisted Living Lifestyles at Scale) programme is a UK-wide digital healthcare initiative that has been designed to support independent living, enhance preventative care, and improve lifestyles by harnessing the potential of e-health technologies and digital services. This short paper presents a brief update on one strand of the University of Glasgow evaluation of the dallas programme. We have used the e-Health Implementation Toolkit (e-HIT) to investigate processes involved in the implementation of e-health tools and digital services being developed and deployed across the dallas communities and to assess 'distance travelled' by communities from baseline to midpoint of a three year programme. Qualitative data analysis was guided by the Normalisation Process Theory (NPT) and Framework Analysis. The e-HIT scores indicated that the dallas communities had underestimated the amount of work involved in implementing at scale. Qualitative data analysis showed that communities have successfully navigated barriers in order to make significant progress in strategic areas, including the development of new models of partnership working resulting in brand recognition and agile service design. The dallas communities are now sharing lessons learned and generating new professional knowledge, skills and understanding across several key strategic areas required for operationalising the implementation of e-health technologies and digital services at scale. The new knowledge being generated through the dallas programme will contribute to the ongoing transformation of digitally enabled healthcare based on more personalised flexible models of provision which resonates with the current e-health policy environment.
\end{abstract}

eHealth, Integrated Delivery of Health Care, National Health Programs, Qualitative Research.

\section{INTRODUCTION}

Healthcare systems across the world face significant challenges due to rising healthcare costs, an aging population and the increased prevalence of chronic diseases. The use of Information and Communication Technologies (ICT) or e-Health is now seen to be an essential component of a modern, cost-effective healthcare system (Department of Health, 2002; Chaudhry et al, 2006).

'DALLAS' is a UK-wide programme for Delivering Assisted Living Lifestyles At Scale (https://connect.innovateuk.org/web/dallas). It is one of the first large scale programmes designed to foster innovation and realise the benefits of digital technologies and e-health services to: (1) enhance independent living, (2) support preventative care and (3) improve lifestyles, all of which will impact on health and wellbeing (McGee-Lennon et al, 2012). Importantly, the dallas programme has a widened scope which includes new models of partnership working across health and care services, business and SME settings in order to operationalise transformational change and contribute to this new and emerging market. The four dallas communities; Living it Up, i-Focus, More Independent and Year Zero are each composed of a large consortium of NHS partners, third sector and voluntary organisations, business and industry partners, academia and government organisations.

Previous studies have shown that implementation and integration of large scale e-health interventions into routine practice is challenging (Sanders et al, 2012). There is an increasing awareness of the need to widen the evaluation of digital health and wellbeing tools and services to take into account socio-technical aspects of large and complex systems including the context of the setting, the intervention itself and the individuals or actors 
involved (Anderson et al, 2008; Boddy et al 2009; Mair et al, 2012).

In the present study, we have used the Normalisation Process Theory (NPT) (May \& Finch, 2009; May et al, 2009) as an underpinning sociological theory to help us evaluate the implementation processes across the four dallas communities. The e-Health Implementation toolkit (e-HIT) (Murray, May \& Mair, 2010) is a well validated toolkit that has previously been applied across a range of e-Health interventions in the UK NHS and more recently, in European countries (MacFarlane et al, 2011). The e-HIT toolkit provides a sensitising tool which addresses three key components involved in implementation: (1) The Context, in terms of the national and local policy and other areas such as leadership and organisational context, (2) The Intervention, tool or service, which in dallas, is a wide array of digital tools and services and (3) The Workforce, which includes professionals, healthcare staff and end users (See Figure 1).

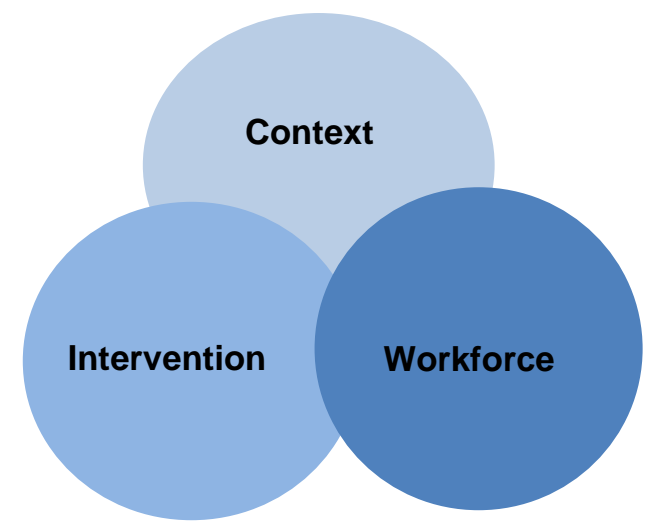

Figure 1: Empirical and theoretical basis of the e-HIT toolkit. (From: Murray, May \& Mair, 2010).

\section{AIM}

We conducted e-HIT evaluation interviews 2 .

The aims of this strand of the dallas programme evaluation were to: (1) Investigate the processes of implementation across the four dallas communities and to assess 'distance travelled' from baseline to midpoint and (2) Report on lessons learned by the communities as the programme has evolved from baseline to midpoint.

\section{METHODOLOGY}

We conducted e-HIT evaluation interviews with a range of dallas stakeholders at baseline $(n=18$; Sept 2012-Dec 2012) and again at midpoint follow up
( $\mathrm{n}=20$; Oct 2013-June 2014) of the dallas programme. Importantly, the stakeholders were drawn from the numerous partner organisations involved in dallas implementation including professionals and project managers from health and social care services, third sector and voluntary organisations, business and SME partners and stakeholders from the technical service suppliers and manufacturers. The e-HIT scores were recorded and analysed using Excel. Qualitative Interviews were digitally recorded, transcribed verbatim and uploaded into the QSR NVivo10 software package for qualitative data analysis. Data analysis was guided by Framework Analysis and NPT (Ritchie \& Spencer, 2002; May \& Finch, 2009).

\section{RESULTS}

Initial findings showed a decrease in e-HIT scores (from baseline to midpoint) for issues related to workload and division of labour, indicating an underestimation of the scale of the work and the level of resources involved in implementation at scale. Qualitative data analysis revealed the significant progress made or 'distance travelled' by all four dallas communities through new models of partnership working which has resulted in progress in key strategic areas such as agile design of digital health products and the importance of developing trusted brand recognition around digital health products:

"European partners and industry players and indeed commissioners in some of our partner organisations ... are....very interested in what we are doing with [name of community] and it aligns very well to some other approaches that are going on in different countries..."

\section{- Dallas Stakeholder /NHS Manager}

Furthermore, communities are reporting on lessons learned from the scale of dallas as well as generating new professional knowledge, skills and understanding required for operationalising e-health tools and digital services at scale:

"Now the thing is implementing something at scale is so different to running a pilot. $[\ldots \ldots \ldots . .$.$] in terms if$ you want to get ahead, if you want to develop something that is attractive to other countries and make an economy out of it in the UK, you've got to get to scale and find out the answer to the questions that nobody else has found out yet....."

- Dallas Programme Manager 


\section{CONCLUSION}

'DALLAS' is one of the first large scale programmes designed to support innovation through the development of technologies as tools and digital services to improve healthcare provision at scale (McGee-Lennon et al, 2012). The dallas communities have made significant progress in implementing new technologies and digital services within the complexity of the current health care environment.

This progress includes insight and understanding related to new partnership models across traditional professional boundary communities such as health service and social care required for large scale implementation in populations (May et al, 2011). The dallas communities have navigated barriers and are now sharing lessons learned as well as generating new professional knowledge skills and understanding across key strategic areas (such as agile digital service design and social innovation) required for operationalising the implementation of e-health and digital services at scale. This new knowledge being generated during the dallas programme will help to inform the ongoing transformation of digitally enabled healthcare provision based on more personalised flexible models of healthcare delivery which resonate with the current e-Health policy environment (NHS England, 2014; Scottish Government, 2012).

\section{ACKNOWLEDGEMENT}

This work was funded by Innovate UK (formerly known as the Technology Strategy Board).

\section{REFERENCES}

Department of Health, (2002). Delivering 21st Century IT Support for the NHS. London 2002, 131.

Chaudhry B, Wang J, Wu S, Maglione M, Mojica W, Roth E, Moron SC, Shekelle PG (2006). Systematic review: impact of health information technology on quality, efficiency, and costs of medical care. Ann Int Med 2006, 16, 144(10): 742-752.

McGee-Lennon M, Bouamrane M-M, Barry $S$, Grieve E, Latina D, Watson N, O'Donnell C, Wyke S, Brewster S, Briggs A, Finch T, Mair F (2012). Evaluating the Delivery of Assisted Living Lifestyles at Scale (dallas). Proceedings of the $\mathrm{HCl}, 2012$. The 26th BCS Conference on Human Computer interaction Workshop available at (http://ewic.bcs.org/content/ConWebDoc/48790)
Sanders C, Rogers A, Bowen R, Bower P, Hirani S, Cartwright M, \& Newman SP (2012). Exploring barriers to participation and adoption of telehealth and telecare within the Whole System Demonstrator trial: a qualitative study. BMC Health Services Research, 2012, 12(1): 220-231.

Anderson, R (2008) New MRC guidance on evaluating complex interventions. British Medical Journal, Research Methods and Reporting, BMJ, 2008, 337: a1937.

Boddy D, King G, Clark JS, Heaney D, Mair F (2009): The influence of context and process when implementing e-health. BMC Medical Informatics and Decision Making 2009, 9: 9-17.

Mair FS, May C, O'Donnell C, Finch T, Sullivan F, Murray E, (2012). Factors that promote or inhibit the implementation of e-health systems: an explanatory systematic review. Bulletin of the World Health Organisation, 2012, 90(5), 357-364.

May C \& Finch T (2009): Implementing, Embedding, and Integrating Practices: An Outline of Normalization Process Theory. Sociology, 2009, 43(3): 535-554.

May C, Mair F, Finch T, MacFarlane A, Dowrick C, Treweek S, Rapley T, Ballini L, Ong BN, Rogers A, Murray E, Elwyn G, Lgar F, Gunn J, Montori VM, (2009) Development of a theory of implementation and integration: Normalization Process Theory. Implementation Science, 2009, 4(29): $9 p$

Murray E, May C, Mair F (2010). Development and formative evaluation of the e-Health Implementation Toolkit. BMC Medical Informatics and Decision Making, 2010, (10): 61-68.

MacFarlane A, Clerkin P, Murray E, Heaney DJ, Wakeling M, Pesola U-M, Waterworth EL, Larsen F, Makiniemi M and Winblad I (2011) Implementation Science 2011, 6: 122-133.

Ritchie J \& Spencer L (2002) Qualitative Data Analysis for Applied Policy Research (2002). Chapter 12 (305-329). In Huberman and Miles (Eds), The Qualitative Researchers Companion. SAGE Publications Inc.

May C, Finch T, Cornford J, Exley C, Gately C, Kirk $\mathrm{S}$, Jenkins KN, Osbourne J, Robinson AL, Rogers A, Wilson R, Mair FS (2011). Integrating telecare for chronic disease management in the community: what needs to be done? BMC Health Services Research 2011, 11: 131 -141.

NHS England (Oct 2014). Five Year Forward View. Available at: (http://www.england.nhs.uk/ourwork/futurenhs/).

The Scottish Government/ NHS Scotland (2012): eHealth Strategy (2011-2017). (Revised July 2012 to include a Sixth Strategic Aim) Edinburgh, 2012. 